\title{
La religiosità immanente nella narrativa di Giuseppe O. Longo
}

Tiziana Piras

\section{(2) OpenEdition}

\section{Journals}

Edizione digitale

URL: http://journals.openedition.org/cei/212

DOI: $10.4000 /$ cei. 212

ISSN: 2260-779X

Editore

UGA Éditions/Université Grenoble Alpes

\section{Edizione cartacea}

Data di pubblicazione: 15 juillet 2009

Paginazione: 221-231

ISBN: 978-2-84310-145-8

ISSN: 1770-9571

Notizia bibliografica digitale

Tiziana Piras, «La religiosità immanente nella narrativa di Giuseppe O. Longo», Cahiers d'études italiennes [Online], 9 | 2009, online dal 15 janvier 2011, consultato il 27 mars 2021. URL: http:// journals.openedition.org/cei/212 ; DOI: https://doi.org/10.4000/cei.212 


\title{
LA RELIGIOSITÀ IMMANENTE NELLA NARRATIVA DI GIUSEPPE O. LONGO
}

\author{
Tiziana Piras \\ Università di Trieste
}

Un compendio significativo della visione metafisica ed etica di Giuseppe O. Longo si trova nel romanzo L'acrobata, dove a causa dei suoi tragici difetti (e soprattutto per la presenza del dolore, manifestazione particolare ma culminante del male) questo mondo è visto come una "prova generale" del mondo vero che verrà. Un demiurgo malvagio o incapace ha creato un mondo imperfettissimo ${ }^{\mathrm{I}}$, tanto da meritarsi il biasimo delle sue creature:

Nessuna forma di riconoscenza, nessuna giustificazione, nessuna indulgenza può esservi per questo terribile sbaglio: l'odio delle creature fu tale che il creatore dovette soccombere. La sua disastrosa creazione tuttavia non perì con lui, proseguì la sua esistenza colpevole con un'inerzia tanto più terribile in quanto è arduo immaginare che cosa possa interromperne il corso, se non la sua progressiva degenerazione: le crepe si allargano, i difetti s'ingigantiscono, il mondo sprofonda in sé stesso. Dalle sue ceneri potrebbe allora, per un atto che non è dato immaginare, e forse neppure sperare, da parte di un principio finora silente, potrebbe sorgere finalmente il mondo nuovo, un mondo che non sia il capriccio di un demiurgo folle e impotente, bensì una creazione assoluta, perfetta, un cosmo che non avrebbe neppure bisogno della materia per esistere in eterno. (Longo, 1994, cap. v, p. 82)

Di questa forte visione di sapore gnostico va in particolare sottolineato il processo di smaterializzazione, che sembra indicare nello spirito una realtà superiore (il titolo stesso del romanzo allude alla leggerezza del

I. Nel romanzo L'acrobata il protagonista, discutendo con l'amico Tommaso la teoria che il mondo abitato dagli uomini sia una "prova generale" di un altro mondo, afferma (Longo, 1994, p. 80): "Io sono convinto che questo mondo non sia l'opera di un essere buono e misericordioso, bensì di un demiurgo cattivo. Alla base della creazione c'è un principio malvagio, la creazione stessa è una colpa, e questo spiega molto meglio di tutte le dottrine proposte finora la colpa dell'uomo, dell'uomo come specie e dell'uomo come singolo individuo. Le colpe degli uomini sono un'emanazione, se non addirittura un'imitazione, della colpa originale che sta alla radice del mondo, la colpa del creatore." 
mondo vero, un mondo che trascenda la corporeità, ma su questo torneremo subito). La superiorità dello spirito rispetto alla materia è affermata insieme all'incapacità della scienza di fornirci il senso della vita:

“È per questo," disse, "che bisogna trascendere la carne e la corporeità. È per questo che bisogna cercare la leggerezza. Perché altrimenti non c'è speranza, la vita non avrebbe alcun senso, come non ne ha per la tua scienza. Per la tua scienza la vita è un tragico scherzo formale, il risultato di migliaia, di milioni di combinazioni aleatorie e fortuite, scavi nella carne e trovi altra carne, non c'è un nucleo trasparente dove si annidi l'essenza vera della vita, per la tua scienza la vita non ha perché, non ha fine, non ha significato, non ha un centro. Forse è vero: la vita non ha senso, e i miei discorsi sul demiurgo ne hanno ancor meno. Ma che cosa ci dà la scienza in cambio di questa disillusione? Quando tutte le questioni di cui si occupa la scienza fossero state risolte, i problemi veri della vita non sarebbero stati neppure sfiorati. La scienza, si dice, ci dà la verità, ma quale verità? Non certo quella di cui abbiamo bisogno". (Longo, I994, cap. vi, p. IO2)

È interessante notare come nell'opera di Longo l'anelito alla trascendenza e alla spiritualità emerga, senza negarlo, da un fondo di corporeità e di pesantezza materica. Nello stesso Acrobata c'è un'esplicita dichiarazione sulla centralità del corpo, ma è un corpo per così dire affinato e sublimato dal mistero che lo avvolge:

Tutto avviene nel corpo, con il corpo e per il corpo, si nasce e si muore con il corpo, con il corpo si soffre e si patisce e si gode, la vita si genera con un atto rapido del corpo [...]. Che le cose più importanti, l'amore, la vita, il nutrimento, la morte avvengano per e nel corpo aggiunge mistero al mistero dell'esistenza. (Longo, I994, cap. viI, p. II7)

Il progressivo riconoscimento dei limiti della scienza come fonte di verità spinge l'autore a ricercare altre sorgenti di assoluto, quell'assoluto di cui gli umani sembrano avere un bisogno incoercibile. Si potrebbe parlare di religiosità, ma è una religiosità, quella di Longo, che ha poco a che fare con le forme della tradizione: è filtrata da una visione sistemica che l'avvicina al panteismo, è passata al vaglio di una razionalità di cui pure denuncia i limiti. Il bisogno di assoluto, sembra dire Longo, ci spinge a cercare senza sosta la fonte del senso profondo della vita, impenetrabile allo scientismo computante. E la fonte del senso è il sacro, inteso come la terra incognita del silenzio e del rispetto, la regione dove si sospende la chiarezza comunicativa esplicita: è il luogo dove gli stolti si precipitano e gli angeli temono di posare il piede, come dice Alexander Pope in un verso, "For fools rush in where angels fear to tread", ripreso poi da un pensatore 
molto caro a Longo, Gregory Bateson ${ }^{2}$. Nella scrittura di Longo a volte il sacro e il senso sembrano celarsi in paesaggi consueti, che subito però si allargano a visioni sconfinate, prolungando un dolore o preludendo a uno struggimento d'amore che sospinge in alto:

Gli prese la mano e lo condusse lentamente per il vialetto fino al cancello. Lui lasciò fare, perché di fronte al regno oscuro del riscatto tutto è incerto, ogni ragione, ogni tempo, ogni calcolo sono vaghi e dubitosi. Giunti al cancello, gli mostrò con ampio gesto la collina disseminata di villette, come se lì, in quelle ondulazioni verdi, ci fosse la spiegazione di tutto e col suo sorriso confortava le svolte della stradina che scendeva verso la città. Ma quel sorriso gli parve stranamente piccolo, insufficiente alla prova che l'attendeva. Nel cielo vi erano dei segni rosati, relitti forse del tramonto, e gli parve che indicassero una serie infinita di trasmigrazioni e di Angeli, al cui termine doveva pur esserci qualcosa. Qualcosa di teso, misericorde e compiuto, come il viso silente di Dio. (La stagione dei viaggi, Longo, 200I, pp. 65-66)

Il silenzio di Dio sembra dirci che il senso della vita consiste proprio nel cercare il senso della vita, ma questo senso non si troverà, se non forse alla fine:

Si è rotto un centro antico che pareva saldissimo, tutto si è frantumato e galleggia nell'aria con una sonnolenza intrisa di spietata dolcezza. Cerco di ricostruire la vita intorno a qualcuno dei frammenti più grossi, mi lego ai rottami come un naufrago. Continuo a non trovare risposte. C'è solo questa lunga catena di persone fatte di carne e di sguardi, che continuano a muoversi a cercare a chiedere. E da un giorno all'altro, da un anno all'altro, tutto, tutto: da via Gualtieri a Trieste, da Ferrara a Parigi, a Pechino a San Francisco. Da un sorriso in una vecchia fotografia a mio padre in divisa con la sigaretta in bocca sulle sabbie della Libia a mio fratello grasso e calvo con tutti i suoi figli alla musica di Mahler... Così negli anni, nei giorni, tutto tutto. (Una semplificazione del dolore, Longo, 2006, p. I8)

Nell'Acrobata il mondo che vediamo è preludio del mondo vero, di cui si predica l'avvento in un futuro imprecisato. All'idea di una doppia realtà dislocata su piani temporali successivi fa riscontro l'immagine di due realtà, disposte, qui e ora, su piani spaziali distinti, di cui una, la realtà sensibile, nasconde l'altra, più vera ma invisibile. La realtà vera non appartiene alla trascendenza, ma è qui, immanente e tuttavia celata dall'altra e s'intravede, a volte, per le crepe e gli spiragli che fendono la realtà sensibile. Tipica è l'immagine ricorrente del "cielo doppio": sotto il cielo che vediamo, ce ne sarebbe un altro, quello vero e tremendo come un urlo:

2. Gregory Bateson (1904-I980) è stato uno dei maggiori studiosi delle discipline che fanno capo al concetto di informazione. Antropologo, sociologo, cibernetico e psicologo, tentò con grande efficacia di tracciare una mappa concettuale che unificasse mente e natura. Giuseppe O. Longo ha tradotto in italiano quattro suoi libri, tra cui il più significativo è Verso un'ecologia della mente. 
Provava una fredda inquietudine, come se dentro il cranio sentisse battere l'ala della follia. Si sentiva prigioniero, vedeva come dal basso un cielo lontanissimo, di un colore azzurro e fermo, un cielo così teso che da un momento all'altro poteva spaccarsi per mostrare una realtà enorme e spaventosa, una realtà accartocciata e tagliente che poteva mangiarsi il mondo in un attimo. (Longo, I998, cap. IV, p. I54)

\section{O ancora:}

Apparve nel cielo mirabile una visione, squarciando le nubi, insostenibile, inarcata sulla terra e sul mare, trionfante, mozzando il respiro. (Longo, I998, cap. v, p. 158)

\section{A volte invece la visione del cielo ulteriore è rasserenante e rappresenta quasi una promessa di beatitudine e di grazia:}

Aveva visto quell'occhio ceruleo qualche mese prima, una sera dopo il temporale, mentre passeggiava nel Giardino Pubblico. Si era aperto improvvisamente verso l'oriente, sopra i colli, mentre dall'altra parte un faticoso tramonto affocava le nubi grigioporpora. Quell'azzurro gli era parso il vestibolo del paradiso, la soglia che poteva condurlo nell'impero della luce, una luce liquida e soffusa, trascorrente dai pianeti come l'acqua dell'eternità. A lungo aveva contemplato quello squarcio, intorno al quale le nubi fluttuavano in una strana e animata immobilità, sfioccandosi e tendendosi come palpebre per conservarne la forma: e dentro, dentro, un'iride lontanissima e trasparente fatta anch'essa di azzurro, ma più intenso e più fondo, che lasciava immaginare un'altezza. Un cielo così Guido l'aveva sempre desiderato. (Longo, I998, cap. VIII, pp. 294-295)

\section{Nella Gerarchia di Ackermann le considerazioni cosmologiche:}

Ha capito, ha capito adesso? Adesso che Le ho detto come stanno veramente le cose, ha capito perché mi voglio svincolare dal tempo e dallo spazio, perché voglio contemplare l'universo, ma non il nostro piccolo miserabile universo, voglio contemplare quello grande, quello che racchiude tutti gli altri, voglio camminare sulla superficie esterna, non sulla fodera del mantello, voglio vedere la luce. Aspetti, aspetti, ecco qua: il principio antropico. Lei si è mai chiesto perché il ghiaccio galleggia sull'acqua? Se il ghiaccio affondasse, la vita sulla terra non sarebbe possibile. E quante coincidenze di questo tipo sono state necessarie perché nascesse l'uomo su questo pianeta, alla periferia della galassia, accanto a una stella insignificante come il sole? Il numero di queste coincidenze è impressionante. L'età dell'universo, la sua velocità di espansione, la formazione del carbonio nelle supernove, la natura delle forze nucleari, il valore delle costanti universali, la costante di Planck, la carica elementare. In questa visione, pensi, l'uomo diventa un principio di selezione per gli universi possibili. Puro caso? Non ci credo, non ci posso credere. L'unica spiegazione è che esistono tanti universi, ciascuno con le sue caratteristiche, ciascuno con le sue costanti, in alcuni c'è la vita, in altri no, in alcuni c'è l'uomo, in altri c'è solo il tirannosauro, in altri solo il basalto. (Longo, I998, cap. VIII, p. 276) 
Sfociano in una ripresa del tema gnostico dei demiurghi, non più malvagi o impotenti come nell'Acrobata, bensì impegnati nella costruzione di mondi incomunicanti:

È la solita storia dei demiurghi che costruiscono il mondo, ciascuno a modo suo costruisce un mondo, ma tra questi mondi non ci sono rapporti, sono mondi incommensurabili. Uno è il mondo in cui viviamo noi, un altro è il mondo di Parsifal e ce ne sono infiniti altri. Passare dall'uno all'altro di questi mondi è difficilissimo, solo a volte, per una di quelle coincidenze che alcuni vogliono attribuire a Dio, si apre una porta, si scorge un paesaggio vertiginoso, un cielo di diaspro solcato da grandi vascelli o da pterosauri di sogno, ghiacciai e foreste, una selva di grattacieli, figurine minuscole che si affacciano con curiosità sul nostro mondo da quella lucida convessità. Quali domande si fanno su di noi quegli esseri, sognano anch'essi di fuggire nel nostro universo, come noi nel loro, per scampare alle miserie quotidiane? Basta essere infelici perché si apra davanti a noi una di queste porte fatali? (Longo, 1998, cap. vIII, p. 276)

Nella Gerarchia Dio compare esplicitamente, ma con caratteristiche antropomorfe e quasi familiari, che gli tolgono qualsiasi alone di trascendenza. Si tratta piuttosto di un alter ego del protagonista, che dialoga con lui con bonaria condiscendenza:

Lo invase una voglia di spazio, involarsi per agevoli strade, incontrare volti di donna, sorrisi, tornar giovane, ecco, riprovare la vita con cauta saggezza, questa volta, chiedere al Signore un'altra prova, che un angelo lo circondasse di ali, lo chiudesse in una bolla protetta dal tempo e lo riportasse indietro.

"Ma per farti tornare indietro," disse il Signore, "dovrei creare un nuovo universo, un universo identico a questo, in cui tu fossi più giovane di vent'anni e però ricordassi ciò che ti è accaduto qui, altrimenti non servirebbe a niente".

"Non lo puoi fare?" chiese Guido.

"E tu, che faresti? Torneresti a Budapest. Parleresti nel ristorante con Farkas. Incontreresti sua moglie. A che punto la tua saggezza e i tuoi ricordi t'imporrebbero di cambiare il corso degli eventi? Accetteresti la chiave da Kühlmorgen? Accompagneresti Eva dal neurologo? Ascolteresti il Castello di Barbablù? Il solco già tracciato sarebbe molto invitante."

E il Signore con un sospiro si affacciò sul mondo. [...] Scosse leggermente la testa, sorrise e tornò a sonare il sassofono con i suoi angeli.

Guido capì che il Signore non l'avrebbe esaudito. (Longo, 1998, cap. IX, p. 310)

Il tema della ricerca del senso pervade anche tutto il primo romanzo di Longo, Di alcune orme sopra la neve, che affronta esplicitamente e con grande finezza il tema del rapporto tra scienza e senso, mettendo ancora una volta in luce i limiti della ragione nella ricerca della verità. Chi si affida alla sola ragione rischia non solo di non cogliere il senso della vita ma anche di perdere la gioia e la rivelazione: 
"Come ti stavo dicendo, un volta che il fisico abbia accettato che l'universo visibile non è fatto né di spirito né di materia, bensì di energia più o meno strutturata, allora è libero di costruirsi una metafisica, ma una metafisica vera, non stravagante e congetturale come quella dei filosofi. I filosofi, della realtà, non hanno mai capito niente... Solo la fisica, la fisica degli ultimi cinquant'anni, o sessanta, ci dà qualche idea precisa sull'universo in cui ci troviamo a vivere." [...] Enrico ascoltava il collega, di qualche anno più anziano, e quelle parole si accordavano con ciò che era venuto imparando negli anni d'università e in quelle prime settimane lì al Centro. Pure era come se le udisse ora per la prima volta nel loro significato profondo: e ciò suscitava in lui, in una zona delicata e sensibile del suo essere, una specie di disagio, o rimorso, faceva rinascere certi ricordi d'infanzia, fatti di nulla, forse, ma che d'improvviso resistevano tenaci all'avanzata rigorosa e trionfale della razionalità. Se veramente, come diceva Fayard, il mondo, e quindi anche la nostra vita, non era che il risultato della rottura di antiche simmetrie che solo le condizioni eccezionali dei primissimi istanti dell'universo erano state in grado di sostenere ma che poi erano crollate nel degradarsi dell'energia e nel nascere della materia; se veramente, come pure diceva Fayard con una frase allusiva e incomprensibile, l'universo non era che un'estrinsecazione del nulla, nel quale poteva in ogni istante ripiombare; se l'unicità di questo mondo non consentiva se non la rappresentazione unica di un atto unico e irripetibile; se questa dolorosa consapevolezza di unicità corrispondeva a un'assenza totale di significato, come Fayard sosteneva ora togliendosi gli occhiali e massaggiandosi lentamente e a lungo col pollice e l'indice la gobba arrossata del naso; se tutto si riduceva a un vacuo incidente provocato da un Dio distratto e impotente; allora non era forse meglio, rifletteva Enrico, rifugiarsi nelle candide fiducie di un tempo, che ponevano l'uomo al centro di una vasta creazione, perfetta e regolata, sotto la mano sapiente e sollecita di un Dio severo e onnipotente e buono e onnisciente e dotato insomma di tutti gli attributi che un Dio vero deve avere? Non era forse più rassicurante credere che questo mondo non è che la copia imperfetta e pallidissima, ma pur sempre eccellente e benigna, dell'altro, in cui i buoni potranno godere le meraviglie del Paradiso in una luminosa eternità priva d'angoscia e di disordine? Ma queste candide ingenuità Enrico non se le poteva più permettere: aveva imparato troppe cose, la sua conoscenza delle leggi della fisica era troppo vasta ormai per consentirgli di vivere, come altri, nel duplice mondo della scienza e della religione, entrambe lontane, entrambe incapaci di esercitare una pressione troppo forte sulla nostra mente e sulla nostra quotidianità. Aveva mangiato il frutto della conoscenza e, dopo, il mondo non poteva più essere lo stesso: doveva fare delle scelte. La vita è veramente un atto unico, che si recita una volta sola in un teatro che non presenta mai due volte lo stesso spettacolo e che, a rappresentazione conclusa, si riempie di un buio totale, invincibile, dal quale non emergono né gli atti di bontà né le lacrime di commozione né gli occhi innamorati con cui ci guardano talvolta le donne, quando la vita canta. (Longo, I990, cap. I, pp. I5-I6)

Ma non c'è solo lo strumento della ragione, la ricerca del senso si può compiere anche attraverso il corpo e il sesso, magari attraverso un'esperienza umiliante, come se anche i contatti più turpi potessero essere fonte di rivelazione. L'apparente dicotomia tra corpo e mente, tra istinto 
e ragione, tra materia e spirito sembra risanarsi in nome dell'importanza fondamentale del mistero e della ricerca:

Lei andò verso il letto e cominciò a spogliarsi, ammucchiando gli abiti sul pavimento sabbioso. Sentiva su di sé gli occhi del guardiano, ma evitava di guardarlo perché temeva di non farcela. Si sdraiò e lui le fu addosso. Mentre lo aiutava, nella mente continuava a risonarle quella serie ininterrotta di domande che non riusciva a formulare e che si traducevano in tanti perché. Era lì, stava facendo qualcosa col corpo, ma le sembrava anche di essere altrove, in una dimensione diversa e più alta e tra $\mathrm{i}$ due luoghi c'erano legami che a lei sfuggivano ma che il suo corpo in qualche modo riusciva a comprendere. "Che cosa significa possedere un corpo?", si chiese ancora mentre l'uomo si muoveva in lei e la schiacciava col suo peso.

Come un animale, pensò, ma poi capì che in fondo non c'era differenza tra il guardiano e Inoue, che tutto era congegnato in un certo modo che non consentiva scampo, che certe forme e certe leggi erano state fissate una volta per tutte. Il guardiano mugolava per un piacere che la stupiva e un poco anche le ripugnava, ma così forse qualcosa avrebbe capito di quella vasta rete di oggetti colori suoni, le dune e il vento e il faro a precipizio sulle onde e lo sguardo fisso concentrico del gabbiano e il treno che adesso portava Inoue a casa, e poi sua moglie, la notte precedente, quelle che sarebbero venute, la folla innumerevole delle città, i canti e gli anni degli uomini, i sorrisi e le morti senza fine lungo la catena perpetua della vita.

"Perché, perché, perché..." le domande adesso uscivano dalla sua bocca in suoni ritmici e convulsi e ottenevano infine una risposta nell'urlo del guardiano che si abbatteva rantolando su di lei, un urlo che veniva dalle profondità del tempo, dalle caverne, dai tiepidi mari della preistoria, dal brulicare indifferente e voglioso del caos. Quella era la risposta della vita, la vita che conteneva lei e il guardiano e Inoue e tutto. (Il Museo del mare, Longo, 1997, pp. 66-68)

Accanto alla ricerca scientifica, c'è dunque un'altra ricerca, che si fa con strumenti diversi dal rigore scientifico, strumenti che includono il corpo e la narrazione, ma che comprendono anche il silenzio e la sospensione. In fondo tra questi strumenti e la razionalità computante tipica della scienza non c'è opposizione quanto piuttosto complementarità, contiguità e operosa collaborazione. E la ricerca sembra inseguire e corteggiare una realtà intima ed elusiva, che non è solo quella soggettiva dell'io, ma è quella misteriosa e soggiacente che avvertiamo esistere nel profondo, una realtà che ci attrae per quel tanto di inspiegato, inspiegabile e indicibile che avvertiamo ovunque: "Perché, in fondo, l'indicibile è l'unica cosa di cui c'interessa parlare." (Ricordo di viaggio, Longo, I995, p. 70)

Si avverte infatti nell'opera di Longo la presenza di una realtà nascosta sotto quella sensibile, pronta a scaturire nei momenti di dolore e di stanchezza, a palesarsi nei punti dove la tessitura del reale si allenta, mostrando fessure, lacerazioni, allentamenti, sfiancature, lacune. Quando ciò accade, 
la realtà ordinaria sembra rivelarsi per ciò che è: una quinta teatrale, un fondale posticcio:

È mezzanotte passata, vado alla finestra e contemplo la solita piazza, i lampioni, le panchine. Questa città sembrava vasta e profonda, invece è sottile come un foglio di carta, le persone sono disegnate, non mi fanno più male. Il brusio sommesso della notte, il mite chiarore, il cielo ondulato di leggerissime nubi, è un vasto teatro animato, una gran macchina, congegni e ingranaggi segreti, quinte e fondali si allargano fino all'Istria, fino al Tagliamento e ancora più in là. (Longo, 1998, cap. x, p. 352)

Questa scoperta, improvvisa anche se presagita, può dare un senso di allucinato straniamento (Isola fortificata, Longo, 1997, pp. I4I-I52) o produrre un esplicito sconvolgimento delle coordinate spazio-temporali (La ferita del tempo, Il fuoco completo, Longo, 1986, pp. 42-47 e 35-4I; Congetture sull'inferno, Longo, I995, pp. 26-35) oppure una confusione di piani con forti dosi di visionarietà (Avvisi ai naviganti, Longo, 200I, pp. 7-3I, oppure il dramma Il cervello nudo, Longo, 2004, pp. 7-67).

Il tema della ricerca del senso è dunque declinato da Longo in diverse forme. Dell'altra realtà non ci sono soltanto nostalgie o presentimenti, ci sono anche visioni più compiute, addirittura la rivelazione piena, come in Angelo a Veronica (Longo, 1995, pp. 57-64), dove tuttavia la rivelazione naufraga nelle pieghe dell'oblio, lasciandosi dietro uno sfarinio di rimpianto. Le enigmatiche parole della rivelazione angelica sono una suggestiva mistura di poesia "statistica" e di termodinamica:

Sciogliendomi dalle inflessibili trajettorie traboccanti d'amore per l'ordine e l'eternità un'alea virtuosa mi ha tratto nel vostro mondo sublunare brulicante della vita che un giorno arbitrario si è raggrumata nell'alfa e nell'omega per generare dai ciclici pozzi del tempo lo sprazzo effimero e ardente della tua desolata coscienza smarrita nel delirante abisso delle molteplici riflessioni di sé stessa che con misurato stupore si chiede come perché tutto e il tremore dei segni recanti da lontananze invincibili il rombo degli universi che si sfaldano nei misericordi fossati del pianto ripetutamente ti allevia d'amore per la tua vita ancora oscillante sulle fornaci del caso. (Longo, 1995, p. 59)

In un'epoca come la nostra, in cui riprende vigore il dibattito sui rapporti tra letteratura e scienza, o, se si vuole ricorrere a una locuzione un po' trita ma ancora efficace, sulle "due culture", l'opera di Longo si distingue. Entrambe fonti di sapere, letteratura e scienza sono tuttavia profondamente diverse quanto al tipo di conoscenza che producono. E ciò resta valido anche se oggi la visione che abbiamo della scienza ha subito cambiamenti profondi, che preludono forse a una convergenza tra le due forme di sapere, quella scientifica e quella letteraria, sotto il comune denominatore della narrazione: ed è questa convergenza che tenta Longo con risultati suggestivi. 
E non è da credere che il narratore Longo dimentichi il suo debito con la scienza: i suoi rigorosi studi di fisica e matematica sono tutti presenti in filigrana. Tuttavia è una presenza filtrata, una sorta di aura o proiezione luminosa che giunge sulla terra da un cielo lontano e impassibile (come il cielo a cui rivolge lo sguardo pieno d'inutile implorazione la protagonista di Aviatore al tramonto, Longo, 2003, pp. 5-22). Ma forse non è un cielo trascendente, bensì immanentissimo, quello che si rivela in una sorta di miracolo e in cui per un istante fuggevole può penetrare lo sguardo indagatore dell'uomo, scoprendo attraverso rare e preziose epifanie la verità ontologica del mondo.

Grazie a questa ipervisione metafisica, che ci è concessa dalla benevola concomitanza di circostanze improbabili, siamo a volte sul punto di cogliere la realtà vera che sta "oltre" la superficie delle cose. È una concezione squisitamente panteistica della natura: il "sacro" è in ogni dove, un sacro che si avvolge nel silenzio, nella luce abbagliante del meriggio, sulle vaste spiagge e nelle distese desolate dei racconti del Fuoco completo (Longo, 1986). È un sacro che chiede rispetto e pazienza, che si può cogliere nell'attesa perseverante, un sacro immanente in tutte le cose, che può disvelarsi in ogni momento a chi sappia interrogarlo con assiduità ma senza insistenza, senza fretta, senza violenza, con un atteggiamento molto diverso dall'irrequieta curiosità dello scienziato. E poi il sacro, rivelatosi per un istante, si dilegua, lasciando un palpito tiepido e commosso, che invita a perseverare nella ricerca, a farne lo scopo della vita.

Non sorretta da una fede codificata e tradizionale, la ricerca di Longo resta vera ricerca, spesso angosciata e angosciosa, quasi sempre solitaria, non guidata da precetti o da verità rivelate. E l'angoscia, giunta a un limite intollerabile, si tramuta, a volte, in leggerezza, in ironia, in sarcasmo. La mancanza di fede, o meglio il rifiuto di ogni ortodossia, si manifesta non attraverso un rozzo ateismo, bensì attraverso un cauto ed esitante agnosticismo, in cui trovano posto aneliti e slanci verso un'ulteriorità che non diviene mai trascendente, ma si incanala piuttosto nel sentimento panico del mistero diffuso. È una religiosità particolare, che inclina al mistero e non si accontenta di soluzioni facili. 


\section{Bibliografia}

I testi citati sono stati tratti da:

Longo, 1986, Il Fuoco Completo, Pordenone, Studio Tesi (seconda edizione riveduta Faenza, Mobydick, 2000). Raccolta di quattordici racconti: Il romanzo circolare; Il gioco del Gundor; Il calcolatore biologico; Le cinque lapidi; Il fuoco completo; La ferita del tempo; Il pianeta del cuore; Il guardiano della Torre; Lo scioglimento dell'enigma; I segni della sera; I giorni giganteschi; Machina dolens; Alle soglie della verità; In un sogno perpetuo.

Longo, 1990, Di alcune orme sopra la neve, Udine, Campanotto (seconda edizione riveduta Faenza, Mobydick, 2007).

Longo, 1994, L'acrobata, Torino, Einaudi, (tradotto in francese da Jean e Marie-Noëlle Pastureau: L'acrobate, Paris, Gallimard, 1996; prix "Laure Battaillon" 1997).

Longo, 1995, Congetture sullinferno, Faenza, Mobydick. Raccolta di quattordici racconti: E finalmente vennero; Una sera di novembre; Congetture sull'inferno; Fantasmi di stoffa; Dopo le esequie; Angelo a Veronica; Ricordo di viaggio; Il silenzio della città; Il sindaco di Riva; La portoghese; Di sera in un vicolo; Il dono della cometa; Dinamica dei fluidi; La piccola Inge.

Longo, 1997, I giorni del vento, Faenza, Mobydick. Raccolta di undici racconti: All'insegna dell'Uomo Armato; I giorni del vento; Il Museo del Mare; Il casellante; I pianeti della stella Polare; Mentre noi, in America...; Le scarpe di ferro; La Casa Gobba; Il Consigliere; Isola fortificata; Le tracce interiori.

Longo, 1998, La gerarchia di Ackermann, Faenza, Mobydick (tradotto in francese da Patrick Vighetti, prefazione di Luisa Ricaldone: La hiérarchie d'Ackermann, Bernin, À la Croisée, 2004).

Longo, 200I, Avvisi ai naviganti e altre perturbazioni, Faenza, Mobydick (tradotto in francese da Daniel Mandagot: Avis aux navigateurs, Bernin, À la Croisée, 2005). Raccolta di dodici racconti: Avvisi ai naviganti; La verità amabile; La stagione dei viaggi; Non mi piacciono le cose troppo dolci; Rosa al confine; Per la greca del Maryland; La moglie del fornaciaio; Con quelle gambe troppo secche; Saturno dal tetto del bunker; Il fiore del viandante; Brasato per tre; A Zenoburg.

Longo, 2003, Prove di città desolata, Faenza, Mobydick. Raccolta di dodici racconti: Aviatore al tramonto; Varani a Comodo; L'aveva rosagrigio; Atlantico, forse; La storia centrale; I sogni viventi; Dune, gabbiani; 
Andavano a sud; Che cosa fare a Denver quando si è morti; Variazioni con boia; Questo lo facciamo dire a Postuma; Prove di città desolata.

Longo, 2006, La camera d'ascolto, Faenza, Mobydick. Raccolta di dieci racconti: Una semplificazione del dolore; Rumpelzimmer; La legge di Ohm; Amuleto; Paesaggio con rovine; Vera Lipanje; Madre; Famiglia; La camera d'ascolto; Gli Svizzeri di famiglia.

Longo si è dedicato anche alla scrittura teatrale, brillante e drammatica, in parte raccolta nel volume Il cervello nudo:

Longo, 2004, Il cervello nudo, prefazione di Ugo Morelli, Rovereto, Nicolodi. Raccolta di quattro drammi: Il cervello nudo; Il casellante; Sulla rotta di Città del Capo; Era una roccia, il colonnello.

Longo ha poi tradotto i saggi di Gregory Bateson:

Bateson Gregory, 1976, Verso un'ecologia della mente (Steps to an ecology of mind), traduzione di Giuseppe Longo, Milano, Adelphi (seconda edizione ampliata, Milano, Adelphi, 2000).

Bateson Gregory, 1984, Mente e natura. Un'unità necessaria (Mind and nature. A necessary unity), traduzione di Giuseppe Longo, Milano, Adelphi.

Bateson Gregory, Bateson Mary Catherine, 1989, Dove gli angeli esitano. Verso un'epistemologia del sacro (Angels fear. Towards an epistemology of the sacred), traduzione di Giuseppe Longo, Milano, Adelphi.

Bateson Gregory, 1997, Una sacra unità. Altri passi verso un'ecologia della mente ( $A$ sacred unity. Further steps to an ecology of mind), traduzione di Giuseppe Longo, Milano, Adelphi.

Ai rapporti fra senso e narrazione Longo ha recentemente dedicato il saggio Il senso e la narrazione:

Longo, 2008, Il senso e la narrazione, Milano, Springer Italia. 
